\title{
Les formes d'adresse dans La fille du charbonnier : distance hiérarchique ou affective? Racha Mohamed Université du Fayoum
}

\section{Introduction}

Dans cette étude, nous nous proposons d'analyser le système d'adresse dans le conte de La fille du charbonnier ${ }^{1}$. En fait, ce système souligne l'insistante intention portée à l'égard du destinataire. Notre attention porte sur le pronom personnel d'adresse et sur les formes nominales d'adresse en vue d'identifier leur nature et leur fonctionnement. Le locuteur à recours à la deuxième personne soit tu, soit vous pour désigner son interlocuteur. Comme le note $\mathrm{C}$. KerbratOrecchioni" lorsque plusieurs formes sont déictiquement équivalentes - comme "tu" et "vous" employé pour désigner un allocutaire unique- elles servent en outre à établir un type particulier de lien social". ${ }^{2}$ L'usage du pronom d'adresse diffère d'une situation à l'autre, d'une personne à l'autre en fonction du niveau de formalité du contexte et des règles en vigueur. Une même personne peut être vouvoyée dans un contexte et tutoyée dans un autre. Le choix de pronom dépend du rapport entre les interlocuteurs. Le tutoiement est lié aux liens de solidarité tandis que le vouvoiement parait plus formel. Alors, ce choix sert à déterminer la relation avec l'interlocuteur et à éclaircir l'image souhaitée du locuteur.

C. Kerbrat-Orecchionisouligne que le choix tu/vous est associé à l'appréciation personnelle et à la prescription sociale : "chaque locuteur en fait son appréciation personnelle, et utilise en conséquence les pronoms d'adresse. Or, cette appréciation personnelle peut à la fois être socialement définie d'une part et révéler beaucoup de l'individualité $\mathrm{du}$ locuteur d'autre part". ${ }^{3}$ L'option pour tu/vous constitue également" un acte d'identité sociologiquement et psychologiquement défini et déterminé par ailleurs par le jugement qu'il se fait de son interlocuteur" ${ }^{4}$. D'ailleurs, le premier à prendre la parole impose le pronom d'adresse, un acte que l'autre peut accepter ou non.

Certaines variables y jouent un rôle significatif comme l'âge, le sexe et le milieu socioprofessionnel des interlocuteurs. Il ne faut pas également négliger des paramètres tels que l'émotion ressentie et la visée du discours. La relation affective influence le choix du pronom d'adresse. La compétence linguistique entre de même en jeu.D'autres facteurs ont également un impact sur le choix de pronom d'adresse comme l'expression faciale (marque de la sympathie ou non), la manière de s'habiller (signe de l'appartenance à un tel milieu), l'aspect physique et plus précisément le comportement plus ou moins hautain et la situation de communication elle-même, notamment le but, le lieu et le temps d'interaction.

J.M. Dewaele formulequ' "étant donné la multitude de variables indépendantes qui déterminent le choix du pronom d'adresse, il n'est pas surprenant d'éprouver des difficultésà

\footnotetext{
${ }^{1}$ M.Mammeri, La fille du charbonnier, Contes berbères de Kabylie, éditions Pocket, 1996.

${ }^{2}$ C.Kerbrat-Orecchioni, Les interactions verbales, tome 2, A. Colin, Paris, 1992, p.50.

${ }^{3}$ Ibid.

${ }^{4}$ J. A. Hughson, ""Tu" et "vous" : étude sociolinguistique dans la banlieue parisienne. Paperpresentedat the colloque sur Les pronoms de la deuxième personne dans les langues de l'Europe, 2003.
} 
maitriser les normes d'usages des pronoms d'adresse". ${ }^{5}$ Le fonctionnement du pronom d'allocution relève de l'ambiguité. C. Kerbrat -Orecchioni met en lumière ce trait qui pose même problème pour un locuteur natif: "les arcanes de la "tu-vousologie moderne" sont décidément bien opaques, et difficilement accessibles au profane...Une telle complexité entraine nécessairement un certain flou dans le fonctionnement de ce système" ${ }^{6}$. En tout cas, le choix du pronom d'adresse est une question délicate qui relève, la plupart du temps d'une affaire personnelle. Cette subjectivité n'est pas étonnante, elle est inscrite dans le langage. Selon E. Benveniste, le langage "se prête à transmettre ce que je lui confie, un ordre, une question, une annonce et provoque chez l'interlocuteur un comportement chaque fois adéquat" ${ }^{7}$.

Certains tel que J. A.Hughson insiste sur la dimension sociale de ce choix. Ce dernier est même pris pour un indice et/ou un symbole des changements sociaux. Les pronoms d'adresse " constituent un paradigme grammatical très efficace pour étudier la relation entre la langue et la société". ${ }^{8}$ Il démontre que "la structure sociale exerce son influence sur le choix que les individus font des pronoms de deuxième personne" ${ }^{9}$ du fait que le langage ne se limite pas à transmettre une information mais le locuteur s'en sert "pour définir (consciemment ou inconsciemment) sa relation avec son interlocuteur, pour construire son concept d'identité à l'intérieur d'un groupe social et pour répondre au contexte social dans lequel il se trouve"10. Le choix d'un tel pronom s'explique en termes de l'appartenance à un tel groupe socio-professionnel. Dans le même sens, D.Maingueneau formule que "les énoncés doivent en effet bien formes non seulement linguistiquement mais aussi socialement"11 et que "s'il est indéniable que le choix de tu ou vous est porteur d'une signification sociale importante, il s'en faut de beaucoup cependant que les notions de "politesse" ou "respect"suffisent à rendre compte de cette signification" 12 .

Tutoyer ou vouvoyer son interlocuteur est-il divergent? En général, les deux pronoms maintiennent une relation évidente avec la situation d'énonciation du fait d'être des formes d'appel personnalisé.Ceux-ci affirment l'importance et le soin accordés au destinataire et son incarnation dans l'acte énonciatif. Ils permettent au locuteur de se situer par rapport à son destinataire et à son énoncé. D.Maingueneu affirme que "l'énonciateur choisit nécessairement un certain mode de présentation de son énoncé, privilégie tel(s) ou tel(s) de ses constituants"13 Pourtant, le vouvoiement suppose une relation d'autorité hiérarchique soit sociale, soit professionnelle.Il s'explique en termes de pouvoir (qu'il soit réel ou feint) et d'insolidarité. Celuici dénote une relation asymétrique et non-réciproqueque le locuteur cherche à imposer. En plus,

${ }^{5}$ J. M.Dewaele, "Vouvoiement et tutoiement en français natif et non natif. Une approche sociolinguistique et interactionnelle" in La chouette, n.33, 2002, p.p.1-13.

${ }^{6}$ C.Kerbrat-Orecchioni, Les interactions verbales, tome 2, A. Colin, Paris, 1992, p.49.

${ }^{7}$ E. Benveniste, Problèmes de linguistique générale 1, Gallimard, Paris, 1966, p.39.

${ }^{8}$ J. A. Hughson, ""Tu" et "vous" : étude sociolinguistique dans la banlieue parisienne. Paperpresentedat the colloque sur Les pronoms de la deuxième personne dans les langues de l'Europe, 2003.

${ }^{9}$ Ibid.

${ }^{10}$ Ibid.

${ }^{11}$ D.Maingueneau, L'énonciation en linguistique française, Hachette, Paris, 1999, p.29.

${ }^{12}$ Ibid.

${ }^{13}$ D.Maingueneau, Approche de l'énonciation en linguistique française, Hachette, Paris, 1981, p.9. 
le degré de formalité perçue dans le vouvoiement est en quelle sorte comme un obstacle empêchant de manifester nettement la colère. En gros, le "vous" est associé au cadre formel, exprime la distance, le respect, la supériorité et la plupart de temps, est imposé et peu naturel.

Quant au "tu", il est réservé au contexte convivial et de partage, exprime la familiarité, l'égalité, la sympathie, la solidarité, l'infériorité, la relation symétrique et réciproque, s'attache aux situations non-formelles et intimes et la plupart de temps, est choisi et plus naturel. C'est un signe d'appartenance à la même sphère discursive et sociale. Il est donc susceptible de produire des effets émotifs. Alors, le tutoiement "n'est pas nécessairement une forme dépréciative (...) tout dépend de la situation de communication, c'est-à-dire des conventions en usage dans le groupe social dans lequel s'inscrit l'énoncé. Il y a en effet bien des cas où le vouvoiement serait marque d'impolitesse, étant interprété comme mise à distance déplacée." ${ }^{14} \mathrm{En}$ outre, la formule non-réciproque tu-vous témoigne d'une forte volonté d'exprimer verbalement le respect envers autrui et souligne la nature hiérarchisée de l'échange.

L'option pour tel pronom s'opère donc en fonction de trois axes : l'axe de la notoriété (personne connue ou non), l'axe de l'autorité (supérieur/ inférieur, égal/inégal), et l'axe de l'attitude (convergent/divergent, approbation/désapprobation, accord/désaccord).Le mélange des pronoms des formules d'adresse dénote une certaine variation de la nature de rapports entre les participants de l'interaction.

DansLa fille $d u$ charbonnier, les personnages sont attribués selon leur rang social (roi, serviteurs, charbonnier) ou selon la relation interpersonnelle (père, fille). Aucun indice de nom propre n'est observé. Dans ce contexte, le pronom d'adresse (vous) est-il un indice fiable, une garantie d'un respect réciproque ou relève-t-il de l'hypocrisie sociale, admise ou imposée? Le jeu du pronom tu/ vous se situe-t-il sur quel axe? En combine-t-il plusieurs? Engendre -t-il diverses formes de relation interpersonnelle?Par quel critère le choix du pronom d'adresse est-il dicté ? Le pronom "vous" indique-t-il seulement un degré de politesse plus élevé que le "tu" ou est-il porteur d'autres valeurs spécifiques? La répartition tu/vous suit-elle un certain schéma? Actualise -t-elle le facteur affectif? Le vouvoiement se manifeste-t-elle seulement dans deux types de relation? Le changement pronominal est-il le reflet d'une perpétuation de la hiérarchisation? Quelle marque affective confère le locuteur à son énoncé et à son destinataire par tel usage pronominal? Y a-t-il un certain écart par rapport aux normes aussi bien sociales que discursives?

Outre le pronom personnel, les formes nominales d'adresse(FNA) ont la fonction de designer, catégoriser et spécifier certaines propriétés de l'allocutaire. Elles sont prises pour une entrée de jeu;elles servent à instaurer le contact avec luiet à marquer une certaine prise de position. Les termes d'adresse se réfèrent à toute forme désignant explicitement l'allocutaire. En effet, interpeler une personne correspond à la fonction phatique de Jakobson. Celle-ci a pour but d'éveiller l'attention de l'interlocuteur et de maintenir le canal de communication ouvert tout en l'encourageant à continuer la communication. La nature même de l'échange, oral et face à face, exige ce système d'adresse afin de garantir l'efficacité de l'interaction. C'est donc une sorte de pression, d'exigence imposée par le discours conversationnel. Selon C.Kerbrat-Orecchioni, "ce sont aussi des outils efficaces pour allouer, réclamer et négocier la prise de parole" ${ }^{15}$. Les formes

\footnotetext{
${ }^{14}$ D.Maingueneau, L'énonciation en linguistique française, Hachette, Paris, 1999, p.30.

${ }^{15}$ C.KERBRAT- ORECCHIONI, "Introduction", in C. Kerbrat- Orecchioni (éd.). S'adresser à autrui: les formes nominales d'adresse en français, Chambéry. Ed. de l'université de Savoie, coll. Langages, 2010, P.P.7-30.
} 
nominales d'adresse(FNA) assurent également "un rôle important dans le fonctionnement des interactions entre autres pour marquer la relation interpersonnelle et construire l'espace social de l'interaction"16. Dans cette étude, nous essayons de répondre à ces questions : quelles sont les formes utilisées et pour quelles raisons? Cherchent-elles à définir ou plutôt redéfinir le statut de la personne interpelée ou à conférer un nouveau aspect socio-affectif?

\section{1-Le pronom d'adresse (tu/ vous)}

Ce conte montre un certain nombre de situations où apparaissent divers personnages dont le statut est diffèrent(le roi, les serviteurs, le charbonnier, l'étranger, les conseillers du roi, la fille du charbonnier ou la reine, le voleur du putain, le chef des serviteurs). Le roi dont le caractère est autoritaire et fantasque, cherche à formuler des énigmes pour accentuer sa supériorité et pour proférer des menaces et ce sont à ses sujets de " les résoudre dans un délai fixé sous peine d'y perdre la vie", "à chaque réponse qu'on lui donnait, il ricanait et disait au malheureux de se mettre de côté". En d'autres termes, il adore poser des énigmes et tuer ceux qui ne savent pas y répondre. D'ailleurs le cadre spatial tel que la cour royale impose un certain emploi du pronom d'adresse. Nous remarquons que le taux de vouvoiement est très élevé dans ce conte.Le tutoiement n'est pas de règle. Le "vous" apparait d'une manière régulière dans les séquences où figure le roi pour ainsi refléter le trait non-réciproque de l'échange et l'impact de la classe sociale sur le choix du pronom d'adresse. Sa présence est certaine dans les scènes où les interlocutoires ne se connaissent pas. Ceux-ci préfèrent se vouvoyer. Alors le facteur connaissance et le décalage hiérarchique entrent en jeu. L'usage non-symétrique de vouvoiement est-il considéré comme déplacé dans certains cas?

\section{1-a .Le "vous" proprement hiérarchique:}

Dans l'interaction roi /charbonnier, nous remarquons que le roi opte pour un "tu" à l'adresse du charbonnier qui vient présenter une solution à l'énigme de roi, tandis que le charbonnier emploie le "vous", marque de politesse, de respect et de distance hiérarchique. En effet le décalage supériorité /infériorité est également signalé par des marques vestimentaires et des termes défavorisés : " un pauvre homme", "tout noir et pauvrement vêtu". La dyade vous/tu se manifeste clairement dans :"-sire, (...) le mot de l'énigme, Dieu seul et vous-même le savez. Néanmoins je pense quant à moi, que votre arbre représente l'année, les branches les douze mois, les rameaux les jours et les feuilles les cinq prières de la journée(...)-tu ne vas pas me dire que tu as trouvé seul le mot de l'énigme". Dans cette réplique,maints outils servent à renforcer la supériorité du roi : le situer au même rang que Dieu grâce à la conjonction de coordination "et", le considérer comme une source suprême et divine d'information. Quant au charbonnier, il insiste par contre à mettre le point sur son infériorité : il s'exclut de la sphère de savoir à l'aide du connecteur" néanmoins", du modalisateur épistémique "pense" et de la locution adverbiale "quant à moi". Ces deux derniers éléments situent logiquement ce qu'il dira par rapport à ses propres connaissances, susceptibles d'être vraies ou non. Nous notons que les eux interlocuteurs emploient le même terme "seul" mais différemment. Le premier sert à rehausser et à honorer tandis que le second, plutôt, à rabaisser et à ricaner.

Cette dissymétrie se maintient encore ("tu" de la part du roi et "vous" de la part du charbonnier): "-qu'attends-tu pour me dire où se trouve cette fille?-Elle est trop jeune...et...de toute façon...indigne de vous". Malgré l'état de perplexe et d'embarras, le charbonnier, sur un ton

\footnotetext{
${ }^{16}$ Ibid.
} 
indécis et en bégayant, réussit à maintenir le même pronom (le" vous"). A la résolution de son énigme. Le roi éprouve à la fois de la colère et de l'étonnement: "s'écria le roi", "le roi se récria". Celui-ci ne parvient pas à croire qu'il y a une autre personne capable, au même titre que lui, de déchiffrer ses énigmes, c'est ainsi qu'il donne ses ordres au charbonnier en utilisant évidemment le "tu" :" tu diras, à ta fille de se préparer". L'association tu+ futur met en lumière le pouvoir détenu par le roi sur le charbonnier, un pouvoir qui lui permet de lui donner des ordres.

La dyade vous/tu est encore remarquable dans l'interaction en justice entre le roi et l'étranger. Le roi est supérieur, c'est lui qui rend la justice. L'étranger est inférieur par le fait de son statut, c'est lui qui demande justice. D'ailleurs, les connotations attachées à ce terme sont généralement péjoratives: manquer de savoirs, être à l'écart, éprouver un sentiment défavorable, ...etc. Le "vous" de l'étranger traduit non seulement une distance sociale et hiérarchique mais plutôt une distance affective parce que celui-ci éprouve de l'amertume, il se sent traumatisé à la perte de sa cause : "vous me voyez réduit à la dernière extrémité". Nous remarquons que le roi insiste à utiliser le "tu" sous ses différentes formes (pronom tonique, adjectif possessif et le pronom sujet) :" encore toi(...) j'ai déjà jugé ton cas hier et tu as été condamné". La figuration d'un "vous" hiérarchique est évidente dans l'échange entre les serviteurs et le roi : "avant de nous donner congé, la jeune fille nous a enjoint de vous répéter ses paroles exactement". Ce "vous" sert à exprimer la distance polie, l'inégalité et le pouvoir. Il s'agit donc d'un "vous" imposé dans ce cercle royal.

Lors de la scène entre la fille du charbonnier et les serviteurs du roi, le recours au "vous" reçoit plusieurs interprétations.Lesserviteurs qui ont prélevé une partie du cadeau envoyé par le roi à sa future épouse choisissent comme signe de respect le pronom d'adresse "vous" :" où est votre père? Où est votre mère? Vos frères?". L'usage de "vous" dans tel contexte est approprié. D'une part il s'agit de la première rencontre, d'autre part, il reflète la supériorité de la fille du charbonnier puisqu'elle sera la future reine. Cette supériorité est accentuéepar ses paroles énigmatiques dont ils se trouvent incapables de comprendre la signification. La fille du charbonnier, en sélectionnant le chef des serviteurs et en lui adressant la parole en "vous", marque la supériorité de sa position par rapport aux autres serviteurs. D'ailleurs, le choix d'un tel pronom confère un trait officiel au message qu'il se charge de transmettre au roi. Par ce procédé, la fille veut également se distancierd'un comportement qu'elle juge indigne: le vol : "dites-lui qu'il manque des étoiles au ciel, de l'eau à la mer et à la perdrix du duvent".

\section{1-b. scène roi/fille du charbonnier(dorénavant la reine)}

Il s'agit de deux scènes. La première se déroule le premier jour de leur mariage, l'autre est à la suite de l'affaire de l'étranger. Nous nous proposons de les traiter en parallèle. Dans la première, nous remarquons l'emploi d'un "tu". Outre le lien familial, les deux interlocuteurs se situent au même niveau quant au déchiffrage de la parole allégorique. Cet usage est donc signe d'égalité et de partage: "le roi, quant à lui, était tout à la joie d'avoir dans son palais une épouse qui pourrait lui donner la réplique et jouer à armes égales avec lui au jeu des énigmes ". C'est elle qui a réussi à résoudre l'énigme du roi (un arbre dont les douze branches portent chacune trente rameaux, chaque rameau produit cinq feuilles).La deuxième personne du singulier assure un effet d'équivalence et d'analogie dans ce contexte.

Ce "tu" semble normal après la célébration de leur mariage. Il dénote une certaine tendance à la réciprocité et à la symétrie. Pourtant, à certaines reprises, il est assez ambigu, dénote-t-il la familiarité et l'égalité ou est-il dicté et imposépar un supérieur à un inférieur? En

(Les formes d'adresse dans La fille du charbonnier : distance hiérarchique ou affective?) Racha Mohamed 139 
effet, dès le premier soir, le roi ne fait que l'avertir et la mettre en garde de peur qu'elle soit un jour au-dessus de lui : "tu es la seule à pouvoir, le cas échéant, me damer le pion. Mais, je t'avertis: je suis le roi et jamais je n'admettrai que ta parole ait barre sur la mienne(...) si cela devait arriver un jour, rappelle-toi bien : ce jour-là sera le dernier que tu auras passé ici, car tu sortiras de ce palais pour n'y plus jamais revenir". Nous notons que le locuteur réagit, ici, en tant que roi qui est en train de signer une sorte de charte inéquilibrée et non pas en tant que mari.Le ton d'avertissement domine sa parole. Plusieurs marques renforcent ce ton : la phrase injonctive, la négation catégorique soulignant le trait définitif de sa décision et l'association tu + futur qui met le point sur le pouvoir du roi sur elle.Un pouvoir qui lui permet de donner des ordres "rappelle-toi".

Après l'affaire de l'étranger, la reine engage la conversation en "vous". S'adresser au roi sur le mode du "vous" impose un certain cadre à l'échange. Il s'adresse plutôt à lui, en tant que roi, responsable de rendre la justice : " vous êtes un juste roi(...) vous avez donné à l'affaire une suite digne de votre équité". Le recours à ce pronom d'adresse parait comme un refuge auquel la reine a recours afin de se protéger de la colère, de la tyrannie et de l'avidité de vengeance du roi. Elle veut de même garder une certaine distance vis-à-vis de l'acte déjà commis auprès de l'étranger et plus précisémentl'idée qu'elle lui a proposée (dire au roi : "le jour où les mules auront des poulains le monde sera détruit" par analogie à "le jour où les poissons sortiront de l'eau ce sera la fin du monde".

Concernant le "vous" du roi, c'estnon seulement pour reprendre en écho le même pronom mentionné par la reine mais plutôt pour annoncer la séparation et affirmer qu'une distance affective est déjà mise en place. Le roi parle sur un ton dépréciatif, sévère et même de menace :"vous me semblez avoir la mémoire bien courte", "avez-vous oublié...", "rappelez- vous","le premier soir que vous êtes entrée dans ce palais". Alors, ce pronom lui permet d'anticiper sur les évènements et de l'exclure de sonunivers d'appartenance. La distance atteint son plus haut degré lorsque le roi lui demande de quitter pour jamais le palais : "faites en sorte que demain, quand je me lèverai, je ne vous voie nulle part dans ce palais". Il est à noter que ce pronom d'adresse est employé d'une manière assez égale entre les deux pôles de l'échange. Dans telle situation de communication, on s'attend à un "tu", dit intime de l'époux/ (se), à un univers non-formel et familier. Cependant, ils se communiquent à la manière des inconnus tout en imposant des barrières et de la hiérarchie à leur relation.

Dans une situation de désaccord et au cours d'une discussion chargée d'émotion, plutôt négative, il arrive parfois que le vouvoiement cède le pas à un tutoiement manipulatoire. Or, ce n'est pas le cas ici. On garde la même forme, le vouvoiement, afin d'insister à exprimer le désaccord et la colère envers une personne donnée, à savoir la reine. Celle -ci maintient également le "vous" et évite de s'adresser directement en "tu" au roi pour plusieurs raisons parmi lesquelles nous pouvons citer :

- Ce pronom convient davantage au caractère tyrannique et autoritaire du roi. Le "tu" ne fait pas plaisir au roi, c'est ainsi qu'elle l'abandonne pour ne pas aggraver et rendre plus complexe le conflit et de peur de créer de nouvelles barrières;

- C'est une façon pour afficher la supériorité du roi tout en suppliant sa grâce;

- C'est un signe de prudence et de prise en garde de la part de la reine; 
- Il affirme provisoirement qu'elle n'a pas réussi à échapper à son sort;

- Le recours à ce pronom fait partie d'une ruse élaborée par la reine pour apaiser sa colère;

- Elle se déguise sous ce pronom, autrement dit, elle fait semblant d'être moins intelligente que le roi. Par ce procédé, elle se montre résignée à l'avertissement déjà formulé par lui : il ne faut jamais qu'elle puisse paraitre plus intelligente que lui. Ce pronom vient donc s'ajouter à la supériorité feinte du roi;

- Elle fait preuve de modestie à l'aide de ce pronom;

- Par ce pronom, elle cherche à le toucher par cette marque de respect;

- Le vouvoiement est considéré comme un procédé visant à retrouver sa confiance et son amour "faites-moi la grâce de venir diner en mes appartements";

- c'est d'une manière ou d'une autre une tentative de s'assimiler à un rang plus prestigieux et plus élevé, auquel elle ne faisait pas partie dans la mesure où " certains rangs sociaux imposaient un vouvoiement réciproque ou non, héritage du pouvoir collectif investi en certains empereurs à une lointaine époque"17

- Dans cette situation conflictuelle, le tutoiement est évité parce que ce dernier équivaut à un mépris pour les partenaires de l'échange. Il relève même du registre d'insulte. Le roi ne tolère pas le tutoiement dans tel contexte;

- L'usage d'un "tu" dans ce cercle familial, égale à une sorte de pression de la part de la fille du charbonnier et de menace quant au roi.

Après l'effet du puissant narcotique, elle garde avec constance le même pronom d'adresse "êtes-vous en état de vous rappeler ce que vous m'avez dit hier?", "mais ce que j'avais au palais de plus cher c'était vous". Le "vous" est donc chargée d'une dimension affective. La reine s'en sert pour intégrer le roi dans sa sphère intime en gardant en même temps ou plutôt en bénéficiant d'un certain ton de respect et d'estime. Alors, le passage du "tu" au "vous" est voulu. Ce changement pronominal va de pair avec un changement affectif. Il est donc inséparable des sentiments ; il manifeste la haine, la colère et l'irritation du roi vis-à-vis de la reine. D'ailleurs, l'influence d'une situation détendue (faire preuve d'être au-dessus du roi ) n'est pas à négliger.

Nous constatons quela relation roi/reine suit le schéma normal de tout conte: une situation initiale, perturbation et péripéties (après l'affaire de l'étranger) et la situation finale où la reine réussit à surmonter les difficultés et les épreuves (menace d'être chassée de palais) grâce à son intelligence. Pourtant, nous avons l'impression qu'il s'agit d'un schéma doublé. Nous trouvons tout d'abord un début pacifique du conte, puis, la présence des formules et des énigmes pour créer du suspens et accélérer le développement des évènements de l'histoire et finalement la résolution des énigmes et le mariage du roi et la fille du charbonnier. Cet évènement sert de

${ }^{17}$ P. Gardner-Chloros, Le développement historique de tu/vous en français et en anglais: parallélisme et divergence. Paperpresentedat the Colloque sur Les pronoms de la deuxième personne dans les langues de l'Europe, 2003. 
nouveau comme un second début qui donne à voir une suite à l'histoire jusqu'à ce que l'on parvient au rééquilibre.La présence de la mêmeformule d'ouverture et de clôture "Machaho" va de pair avec la forme circulaire du conte.La fin correspond au manichéisme et à la classification des bons/des méchants et que ce sont les bons qui aident les gens et gagnent à la fin. C'est là la morale véhiculée et la conclusion tirée. Cette fin fait valoir le modèle de la fille du charbonnier, un exemple à suivre et à être gardé en mémoire, en particulier au niveau du maniement de l'usage du pronom d'adresse.

\section{1-c.Vous: distance physique et affective}

Nous observons un usage particulier de "vous" dans la conversationoù l'un raconte les péripéties de sa mésaventure à un autre à la porte de la ville : le vol de son poulain et la perte de sa cause en justice. Entre le voleur de poulain et l'étranger, un "vous" s'impose afin de montrer que c'est la première rencontre et qu'ils ne se connaissent pas. Ce pronom sert davantage à dénier le vol"je ne sais pas de quoi vous parlez(...) ce poulain est né de la mule que vous voyez ici et tous les deux sont à moi". Cet usage est considéré comme une stratégie visant à dissimulerla fourberie du voleur.

Dans la même scène, le "vous", revêtu cette fois d'un aspect d'hypocrisie, figure entre ce voleur et le roi : "votre justice est là pour défendre les innocents(...)en votre ville, où je savais pouvoir commercer librement, sous la protection de vos justes lois, reconnues jusque chez vos ennemis". Dans ce discours élogieux vantant les avantages de son règne et de son royaume, le "vous" est associéà des termes intentionnellement affectifs qui font plaisir au roi et qui font appel à des traits personnels mélioratifs tels que "votre justice, vos justes lois". Le voleur fait encore preuve d'hypocrisie dans "votre ville" où il considère la ville comme une propriété réservée seulement au roi. Ces techniques l'aident à faire semblant d'être honnête.

La reine en s'adressant à l'étranger opte tout d'abord pour un "vous" parce que c'est difficile de tutoyer un inconnu. En plus, le fait de ne pas avoir tant de connaissances concernant son âge, sa profession,... rend beaucoup plus approprié le vouvoiement. Ce choix met également le point sur la distance physique qui les sépare : "vous n'avez pas besoin de me voir(...) l'essentiel est que vous m'entendiez et que vous vous conformiez très exactement à ce que je vous direz". Pourtant, le taux de vouvoiement baisse très rapidement, la reine passe à un "tu". Ce passage pourrait être interprété de différentes façons :

-signe de sympathie envers sa cause :"le cœur de la reine se soulevait d'indignation" et reflet d'une forte volonté à sauver un homme que le roi a condamné; ce qui confère à la reine la figure d'un "sauveur". Aider l'étranger se présente en tant que devoir que la reine se met dans l'obligation morale d' effectuer. Cet engagement se manifeste à travers l'association je + futur "je vous direz". Il s'agit d'une sorte d'intégration affectivedans la dynamique discursive et d'une sorte d'invitation à l'action tout en ayant encore de l'espoirLe recours à ce pronom fait partie d'une stratégie visant à légitimer la position de l'étranger et même toute autre position semblable et à dénoncer toute forme d'injustice due à une autorité irresponsable. Ce point de vue va de pair avec celui de D.M" parler, c'est obligatoirement interpréter, apprécier voire juger" ${ }^{18}$.

-signe de la distance hiérarchique; la reine se présente en position supérieure grâce à son statut social et à son savoir. C'est elle qui propose la solution à sa malheureuse aventure :" tu n'as

${ }^{18}$ D. Maingueneau, Nouvelles tendances en analyse du discours, Hachette, Paris, 2001, p.382. 
pas su défendre ta cause", "en faisant ce que je vais te dire". La reine se met à prescrire les étapes à suivre. Elle assigne donc à elle-même et à son allocutaire un certain rôle.

La reine poursuit l'échange en "tu", cette utilisation favorise plutôt la seconde hypothèse. Il faudrait également tenir compte de l'effet de l'usage du tutoiement: suggérer la proximité, l'équité et la justice sociale. Mobiliser de telles valeurs est capable de mener à l'action. Quant à l'étranger, éprouvant de la gratitude et de joie, il maintient le "vous" : "vous me sauveriez la vie".Autrement dit, celui-ci est obligé de continuer à la vouvoyer signe de respect et de reconnaissance.Alors, le passage d'un pronom d'adresse à l'autre est dans ce sens voulu et intentionnel. Ce changement est plutôt pour des raisons affectives. Le changement voulu et nonréciproque du pronom d'adresse est dû au degré de connaissance et à l'impact de la situation dont il est question. Ce jeu pronominal oscille entre éloignement et rapprochement.

Dans ce conte, le choix du pronom allocutif est porteur d'un message précis et reflète les normes en vigueur. C'est comme le signale D. Maingueneau "l'emploi de tu ou vous n'est donc pas univoque et doit être rapporté à des contextes sociaux déterminés" ${ }^{19}$. En outre, les contraintes du genre, le conte, ont leur mot à dire: " dès qu'on aborde le domaine des différents types de discours on a nécessairement affaire à des systèmes plus ou moins lâches de contraintes spécifiques sur l'emploi des personnes" ${ }^{20}$. Il n'est pas aléatoire, il nous informe sur la nature de relation entretenue entre les interlocuteurs. À un niveau hiérarchique, letutoiement est exclu tandis que le pronom d'allocution, vous, parait comme une marque de distance, de réserve, de respect imposé et de pouvoir réel ou symbolique.Ce choix équivaut à une sorte de contrat signé entre les deux interlocuteurs. Le passage du tutoiement au vouvoiement affirme la colère éprouvée par le roi envers la reine tandis que le changement de "vous" en "tu " reflète surtout la sympathie envers une plainte. L'influence de la situation dé/tendue est à prendre en considération afin d'opérer un choix entre tutoyer ou vouvoyer autrui.

\section{2- Les formes nominales d'adresse (FND)}

Outre la forme pronominale du système de l'adresse, il y a encore la forme nominale en tant qu'activité d'adressage. Tout au long de ce conte,nous ne notons aucun indice de prénom. Tous les personnages sont interpelés en fonction de leur statut familial, social ou professionnel. Ce procédé vient s'ajouter à la dimension hiérarchique puisque le prénom sert généralement à diminuer la distance entre les partenaires de l'interaction.T.Johnen note que les FNA "sont sémantiquement plus riches que les formes pronominales, permettant d'exprimer des valeurs relationnelles et sociales plus nuancées. Elles sont corrélativement chargées de fonctions pragmatiques diverses" ${ }^{21}$. De même, l'acte d'adresse contribue davantage à établir le contact au même titre que l'acte de salutation, à susciter une action réactive de la part du destinataire. D'ailleurs, en mentionnant le titre, la profession ou n'importe quel attribut de l'autre pôle de l'échange, le locuteur cherche à renforcer et à intensifier le contact communicatif. Ainsi, cet acte

\footnotetext{
${ }^{19}$ D.Maingueneau, L'énonciation en linguistique française, Hachette, Paris, 1999, p.30

${ }^{20}$ Ibid, p.31.

${ }^{21}$ T. Johnen, "Les FNA en français et en portugais: considérations théoriques et analyses fonctionnelles dans des débats médiatiques électoraux au Brésil, au Portugal et en France" in C.Kerbrat-Orecchioni (éd.) s'adresser à autrui : les formes nominales d'adresse dans une perspective comparative interculturelle, Chambéry. Ed. de l'université de Savoie, coll. Langages, 2014, P.P.375-416.
} 
est "un acte de langage par lequel le locuteur manifeste une certaine considération vis-à-vis des personnes mentionnées dans l'énoncé" ${ }^{22}$.

Il parait également indispensable de tenir compte de l'intention cachée derrière cet acte qui exige une réaction verbale ou non-verbale : "un acte d'adresse peut déclencher au moinsun acte réactif mental chez l'allocutaire, mais éventuellement aussi un acte réactif non-verbal (...) ou un signal d'écoute" ${ }^{23}$.Ces formes contribuent à l'assimilation de l'information transmise. Elles "facilitent la segmentation informationnelle. Cela permet non seulement de relier l'allocutaire au contenu de l'énoncé(...) mais cette segmentation favorise également le processus de traitement cognitif d l'information". ${ }^{24}$ Finalement, elles jouent un certain rôle dans la gestion des tours de parole.

Dans le conte, objet de notre analyse, quelques questions s'imposent : en quel moment de l'interaction figurent-elles? Accompagnent-elles quels actes de langage? Assurent -elles à la fois une fonction déictique et définitoire? Les formes nominales d'adresse : "qu'elles constituent l'allocutaire en tant que tel, qu'elles le sélectionnent parmi un ensemble de candidats possibles, ou qu'elles le confirment simplement, ces formes signifient toujours que "c'est à toi /vous que je parle prioritairement". ${ }^{25}$ En général, les termes d'adresse se caractérisent par la propriété de détachement au niveau de la syntaxique et la prosodie. Ce trait confère plus de saillance à ce qui est énoncé. Ils sont également accompagnés par des éléments non-verbaux: contact oculaire, orientation et direction de corps vers lui. Les possibilités combinatoires sont notables dans certains cas comme ceux destinés au roi. En principe, nous distinguons deux formes d'adresse. L'une est directe, l'autre est Indirecte (une personne présente est traitée comme une tierce personne à l'aide de la troisième personne). Cependant, dans La fille du charbonnier, ce sont les formes directes qui sont massivement utilisées. Certaines formes, surtout les labels et les noms de métiers, y sont réservés à un emploi particulier.

\title{
$\underline{\text { 2-a. Plusieurs formes appellatives }}$
}

\section{- Les noms de métiers et de fonction}

Le roi, grand amateur d'énigmes, en s'adressant au charbonnier, il insiste à l'interpeler en termes de son métier, nomination en fonction de sa fonction sociale:"Et toi, l'homme au charbon, qu'est-ce que tu as trouvé?", "Charbonnier, tu as sauvé ta tête et celle de tous tes compagnons, car tu as touché juste". En fait,gommer les hommes à des fonctions est en quelque sorte une manière pour les mettre au-dessous de leur valeur réelle et les ramener à un degré inférieur. La forme d'interpellation, ici, sert également à sélectionner un allocutaire bien précis parmi l'entourage du roi. En plus, dans le cas de l'énoncé souligné, elle accentue la pression exercée par le locuteur sur l'allocutaire et exige une réaction verbale bien précise.Elle a donc pour effet de renforcer l'impact de l'énoncé.

\section{2-Des titres honorifiques}

\author{
${ }^{22}$ Ibid. \\ ${ }^{23}$ Ibid. \\ ${ }^{24}$ Ibid. \\ ${ }^{25}$ C.Kerbrat- Orecchioni, "Introduction", in C. Kerbrat- Orecchioni (éd.). S'adresser à autrui: les formes nominales \\ d'adresse en français, Chambéry. Ed. de l'université de Savoie, coll. Langages, 2010, P.P.7-30.
}


Il s'agit d'une forme simple, un seul lexème :"sire". Ce dernier sert àétablir une relation particulière à la fois affective et hiérarchique.Il se réfère, tout au long du conte, à un même interlocuteur, à savoir le roi.C'est la forme adaptée à la situation et à la nature de la relation interpersonnelle : supérieur/ inférieur. Elle est proférée par divers locuteurs:

- le charbonnier " Sire, le mot de l'énigme, ...", " Il est vrai, Sire"

- l'étranger ou la victime : "Sire, vous me voyez réduit à la dernière extrémité(...)Sire, je n'avais rien d'autre et je viens porter plainte devant votre justice", "Sire(...) je demande grâce à votre majesté, car ce n'est pas pour l'affaire d'hier que je viens", "Sire, j'ai planté près de la rivière...", " mais, Sire, de grâce, ne dit-on pas aussi que le jour où les mules auront des poulains les temps seront révolus?", "Sire, une voix du ciel me l'a inspirée". Nous remarquons que l'étranger insiste à utiliser cette forme d'adresse comme c'était le seul stratagème qui garantit le gain de son procès et rétablit la vérité. Il opte également pour les mêmes termes proférés par son adversaire: "votre majesté, votre justice".

- le voleur de poulain: "mais, Sire, si votre bonté permet que j'avance une suggestion, il y a peut-être un moyen de trancher".

- le chef des serviteurs: après la remise du cadeau du roi à la fille du charbonnier: "Sire, nous vous rapportons très exactement les paroles de la jeune fille". Cette interpellation sert de preuve de crédibilité. Le chef des serviteurs, au nom de tous les serviteurs, cherchent à jurer devant lui. Dans "assurément, mais, Sire, nous n'avons rien compris", le terme d'adresse atteste de son infériorité par rapport à la supériorité du roi quant à la compréhension de l'étrange partage du poulet : le père/la tête- la mère/le dos-les sœurs/les ailes- les frères/la poitrine- les serviteurs du roi/les pattes.Le roi réussit à comprendre la parole développée de la jeune fille, ce qui rend les serviteurs stupéfiés et émerveillés. II éclaircit ce partage : le père est la tête de la famille; la mère sur elle repose le poids de toute la maison; les frères sont les défenseurs; les sœurs se marient et s'en volent;les serviteurs, sur leurs pieds qu'ils sont allés jusqu'à elle et qu'ils devaient repartir.

- la reine, comme tout autre inférieur, a recours à la même forme d'adresse "Sire" afin de demander la grâce et le pardon du roi et d'apaiser sa colère :" Sire, (...) puisque je connais la disgrâce de vous avoir déplu, puis-je vous demander de m'accorder une faveur dernière?". Cette forme ne fait que mettre le point sur la distance instaurée entre ce couple. La forme nominale d'adresse sert comme "un marqueur de dérivation illocutoire, servant à confirmer, voire constituer la valeur de requête d'un énoncé formellement assertif" 26 : "Sire(...)si vous le permettez, nous allons tout garder dans cette maison, car tout ce que j'ai emporté, c'était pour votre service".

\footnotetext{
${ }^{26}$ C.Kerbrat- Orecchioni, "Introduction", in C. Kerbrat- Orecchioni (éd.). S'adresser à autrui: les formes nominales d'adresse en français, Chambéry. Ed. de l'université de Savoie, coll. Langages, 2010, P.P.7-30.
} 


\section{3-Les labels}

Selon C.Kerbrat- Orecchioni, cette catégorie fait " un catalogage de l'interlocuteur, soit en l'insérant dans une sous-classe(...) soit en le désignant par synecdoque ou métonymie à partir d'une caractéristique jugée particulièrement saillante"27. Nous remarquons cette technique d'adresse dans :

- "Misérables! Qu'avez-vous fait de mes présents?", cette appellation, suggérant un malheur lamentable, décrit l'état de ses serviteurs après la découverte de leur vol, annonce la punition qu'ils subiraient et exprime la colère du roi vis--à- vis de leur conduite malhonnête.

- "Tout n'est pas perdu, étranger", la forme d'interpellation, mise en usage par la reine, est en fonction de son statut.C'est également le terme dont il s'attribue lui-même et sur lequel il met le point : "où je suis étranger", "si bien que me voilà étranger, seul et à peu près complètement démuni".Cette appellation de la part de la reine sert à commencer l'échange et à établir le contact avec lui tout en éprouvant de la pitié pour sa cause.

- La même appellation, étranger, de la part du roi sert d'un côté, à compenser la faute déjà commise à son égard, une sorte d'excuse symbolique : "il est vrai, je l'avais oublié et je te remercie, étranger, de m'en avoir fait souvenir". De l'autre côté, elle est considérée comme une sorte d'avertissement et de menace "te rends-tu compte, étranger, que tu es en train de blasphémer?"

\section{2-b.Normes d'usage}

Nousconstatons que le terme d'adresse, en l'occurrence "sire" est en concomitance avec le pronom d'adresse "vous" pour mettre le point sur sa supériorité. Pourtant, il y a parfois des formules elliptiques où il n'y a ni pronom ni termes d'adresse. Comme c'est le cas dans: "il y a encore autre chose, dit le chef des serviteurs. -Et quoi?". Dans cet échange, la priorité est plutôt accordée au contenu de l'énoncé.

L'interpellation" sire" est accompagnée par des tournures marquant la politesse et des expressions d'un sentiment favorable : "si vous le permettez, si vous le désirez. J'aurai grand plaisir". Celles -ci intensifient l'acte d'adresse. Ce titre sert d'étape préliminaire avant d'engager la conversation avec le roi. Il figure avec une certaine extension sous la forme de "votre majesté". Quant au titre professionnel" homme au charbonnier", le roi s'en sert afin d'affirmer sa propre supériorité. En ce qui concerne la reine, en choisissant l'épithète "étranger", elle se montre respectueuse et sympathique pour sa cause, cette valeur va à l'encontre avec celle attribuée par le roi à l'adresse de la même personne. Le roi ne fait que mettre en avant et réactiver constamment sa supériorité statutaire. Il y a donc une corrélation entre les formes d'adresse en usage et l'image de soi revendiquée. En fait, "tout échange

\footnotetext{
${ }^{27}$ C.Kerbrat- Orecchioni, "Introduction", in C. Kerbrat- Orecchioni (éd.). S'adresser à autrui: les formes nominales d'adresse en français, Chambéry. Ed. de l'université de Savoie, coll. Langages, 2010, P.P.7-30.
} 
linguistique apparait comme une subtilenégociation pour sa face positive et sa face négative; ce qui ne peut se faire qu'en ménageant les faces de son interlocuteur"28.

Ces formes figurent soit en tête de l'énoncé, soit en fin d'énoncé. La première position contribue à attirer l'attention de l'allocutaire tout en le désignant explicitement et le montrant comme le seul à qui la parole est destinée et à mettre le point sur le type de relation entretenue au cours de l'échange. La seconde vise à renforcer l'acte de langage qui l'accompagne : assertion, reproche, menace, ...etc. et à mettre également en lumière le lien social et affectif entre les deux interlocuteurs. Cette FNA fait partie de la mise en valeur du roi. Le titre "sire", seul ou en combinaison avec des termes à forte teneur affective, manifeste un respect particulier à l'intention du roi. Elle s'insère au moment approprié. Elle constitue même la norme lorsque l'on s'adresse au roi. Celle-ci témoigne, d'une certaine manière, de la connaissance et de la maitrise de bien parler. Autrement dit, elle montre une certaine compétence, une certaine familiarité de l'art de s'adresser à autrui.Les titres génériques comme monsieur /madame et les prénoms sont exclus de ce conte. Alors, les formes d'interpellation assurent une fonction linguistique de désignation et de marquage socioaffectif. Celles -ci s'articulent sur deux axes, l'un est à valeur positive (sire), l'autre est à valeur négative (misérable, étranger).Elles correspondent donc à des considérations stratégiques.

\section{2-c. Leurs fonctions}

L'analyse de ces diverses formes d'adresse donne à voir des fonctions variées. Nous pouvons les récapituler ainsi :

- Dénoter la politesse, la distance et le respect;

- Montrer que le locuteur a un savoir préalable sur son allocutaire;

- Utiliser certains titres indique une relationetun savoir personnifiés de l'allocutaire. F.Neveu note que la FNA construit "une cellule référentielle et informationnelle qui enrichit la désignation, la complexifie en lui conférant des capacités présuppositionnelles" ${ }^{29}$;

- Garantir une forte " accessibilité référentielle" 10 et assurer une fonction de rappel surtout lorsque le même terme d'adresse se répète, comme c'est le cas pour "Sire";

- Etre chargées de maintes valeurs pragmatiques en fonction de l'acte de langage auquel elles se trouvent associées. Elles ne sont pas neutres.Si elles sont attachées aux rituels de politesse, elles servent à mettre le point sur le caractère poli de l'énoncé et de l'énonciateur.

- Jouer un rôle important dans la gestion des tours de parole et sélectionner un allocutaire bien déterminé;

\footnotetext{
${ }^{28}$ D.Maingueneau, L'énonciation en linguistique française, Hachette, Paris, 1999, p.31.

${ }^{29}$ NEVEU F., "Détachement et connexité", inSyntaxe et sémantique, n.8, 2007/1, p.p.165-178.

${ }^{30}$ Ibid.
} 
- Contribuer au bon accomplissement d'autres actes de langage, en particulier la requête et l'affirmation; être au service du renforcement de l'acte principal de l'énoncé;

- Catégoriser la nature de la relation interpersonnelle;

- Définir et/ou réactualiser un certain ethos et identité sociale;

- Régler et moduler la distance interlocutive; à la fois proche et lointaine dans certains cas;

- Véhiculer une certaine dimension affective surtout dans des moments de tension afin d'affirmer la réfutation d'un tel acte, en l'occurrence le vol du cadeau du roi;

- Marquer le décalage supériorité/ infériorité entre locuteur /allocutaire;

- Rendre l'allocutaire responsable d'un élément du discours, notamment pour la forme d'adresse "misérables";

\section{Conclusion}

Nous remarquons que le locuteur a le choix entre deux pronoms d'adresse" le "tu" et le "vous". Ce choix est conditionné par la dimension hiérarchique, et le facteur affectif. Il est porteur d'une signification sociale. Les notions de politesse et de respect entrent en jeu. Le locuteur se donne à lui-même un tel statut social et en alloue un autre à son allocutaire. Utiliser tel ou tel pronom s'avère majoritairement important pour préciser la nature de relation. L'usage de "vous" est dépourvu de l'expression de l'ironie dans ce conte. Aucune marque d'hésitation quant à l'usage de tel pronom d'adresse n'est notée. D'ailleurs, la technique d'évitement au niveau d'adresse, par le recours au pronom "on", ne figure guère. Dans la relation familiale (roi/reine), le "vous" est porteur de sens social et affectif. Autrement dit, le pronom de la deuxième personne du pluriel, marque l'attitude, favorable ou défavorable, du locuteur envers son interlocuteur.

Divers facteurs interviennent lors du choix d'un tel pronom : la prise en considération des rapports hiérarchiques, le degré de connaissance, ... etc. L'analyse de ce conte donne à voir que la corrélation entre vous et respect est erronée et que le vouvoiement est une marque de distanciation sociale et affective. Pour le roi, le "vous" marque l'exclusion de la personne vouvoyée, la reine en l'occurrence, de sa représentation familiale et reflète un comportement hautain de sa part et face auquel la fille du charbonnier n'a qu'à lui répondre en écho.

Le "vous" contribue à préciser le type de la relation interpersonnelle soit horizontale (mettre en place une certaine distance), soit verticale (marquer une relation hiérarchique, relation de pouvoir, de supérieur à un inférieur). D'ailleurs, il illustre le caractère conflictuel de la relation, notamment celle de roi /reine. Par le choix de ce pronom, la dimension affective s'éclipse entièrement en situant les partenaires de l'échange dans une hiérarchie sociale, négligeant complètement la dimension familiale.

Entre les serviteurs ou ceux qui viennent porter plainte et le roi, le vouvoiement nonréciproque est de règle. En gros, le "vous" est un synonyme de distance respectueuse et de réserve, une manifestation de la hiérarchie sociale et un outil favorisant le respect des serviteurs à l'égard du roi. C'est donc un usage obligatoire dans ce cercle royal où l'isotopie du pouvoir en est la norme. Entre le couple, on s'attend à un tu, signe d'affection et d'intimité, pourtant, le "tu" donne place au "vous", il marque plutôt la dégradation et la distance affective. Autrement dit, il va de pair avec une émotion d'amour inaccrue. Ce n'est que le prélude d'une séparation. Le 
pronom d'adresse allocutif choisi convient davantage à la force, même feinte, attribuée au roi dans tel contexte. En outre, la fille du charbonnier reprend en compte les barrières sociales qui existées déjà entre elle et le roi. Alors, l'option pour le "vous" est attaché au type de relation interpersonnelle et au degré de la sympathie mutuelle. Elle véhicule donc une tendance affective.

Dans ce conte, les termes d'adresse occupent une place assez importante, surtout dans les séquences d'échange dissymétriques et hiérarchiques. Ils se manifestent d'une façon moins aléatoire, dans des moments stratégiques de l'interaction. Ils sont chargés de diverses valeurs sociales et relationnelles. L'emploi phatique de ces termes parait courant dans ce conte. Les formes attestées sont variées au niveau de la construction, de la valeur et de l'effet allocutif.Plusieurs formes sont mobilisées. Elles sont pourvues d'une relation socio-affective et sont indispensables dans le fonctionnement et la gestion de l'interaction. Elles identifient également le type de lien interactif et l'acte de langage qui les accompagne. La plupart de temps, ellessont doublées d'une autre valeur illocutoire, à savoir l'avertissement, le reproche et la surprise. L'insistance à utiliser "sire" sert à (re)valoriser ce titre. Les FNA servent également à distinguer un destinataire bien précis parmi d'autres et solliciter son attention comme c'est le cas dans le cadre royal. Elles figurent en principe où il y a le roi soit locuteur, soit allocutaire.

Les formes d'adresse, soit pronoms, soit termes, font partie intégrante du savoir linguistique, social et culturel. Ainsi, sont-elles un signe visible d'une telle identité et manifestent-elles les caractéristiques d'une telle communauté sociolinguistique.

Bibliographie

-BENVENISTE E., Problèmes de linguistique générale 1, Gallimard, Paris, 1966, -COFFEN B., Histoire culturelle des pronoms d'adresse. Vers une typologie des systèmes allocutoires dans les langues romanes, Champion, Paris, 2002.

- DEWAELE J. M., "Vouvoiement et tutoiement en français natif et non natif. Une approche sociolinguistique et interactionnelle" in La chouette, n.22, 2002, p.p.1-13.

- GARDNER-CHLOROS P., "Le développement historique de tu/vous en français et en anglais: parallélisme et divergence", Paperpresentedat the Colloque sur Les pronoms de la deuxième personne dans les langues de l'Europe, 2003.

- $\quad$ "L'usage de tu/vous, un acte d'identité?" Paper presented at the French Language and questions of identity, 2002.

- $\quad$ " Ni tu ni vous : principes et paradoxes dans l'emploi des pronoms d'allocution en français contemporain " in Journal of french languagestudies, n. 1(2), 1991, p.p. 139-155.

-HAVU E., SUTINEN J., "L'emploi des termes d'adresse dans le français parlé du film. Comparaison avec une enquête sur questionnaires, 2005, Stockholm, Suède, p.p.289-302

- HUGHSON J.A., ""Tu" et "vous" : étude sociolinguistique dans la banlieue parisienne. Paperpresentedat the colloque sur Les pronoms de la deuxième personne dans les langues de l'Europe, 2003.

-JOHNEN T., "Les FNA en français et en portugais: considérationsthéoriques et analyses fonctionnelles dans des débatsmédiatiquesélectoraux au Brésil, au Portugal et en France" in C.Kerbrat-Orecchioni (éd.)s'adresser à autrui : les formes nominales d'adresse dans une 
perspective comparative interculturelle, Chambéry. Ed. de l'université de Savoie, coll. Langages, 2014, P.P.375-416.

2014

- KERBRAT-ORECCHIONI C., "Introduction", in C. Kerbrat- Orecchioni (éd.).S'adresser à autrui: les formes nominales d'adresse en français, Chambéry. Ed. de l'université de Savoie, coll. Langages, 2010, P.P.7-30.

- $\quad$ Les interactions verbales, tome 2, A. Colin, Paris, 1992.

- KHEDER F., Les termes d'adresse en français contemporain, Thèse de doctorat, Paris: Université de Paris 3, 2004.

-LAGOGETTE D., Termes d'adresse, insultes et notion de détachement en diachronie: quel critère d'analyse pour la fonction d'adresse? Cahiers de praxématique, n. 40, p.p.43-70.

- MAINGUENEAU D., Nouvelles tendances en analyse du discours, Hachette, Paris, 2001.

- $\quad$ L'énonciation en linguistique française, Hachette, Paris, 1999.

- $\quad$ Approche de l'énonciation en linguistique française, Hachette, Paris, 1981.

- MAMMERI M., La fille du charbonnier, Contes berbères de Kabylie, éditions Pocket, 1996.

-MAURER B., "Quelques méthodes d'enquête sont effectivement employées aujourd'hui en sociolinguistique" in L.J.Calvet et P.Dumont (eds.)L'enquêtesociolinguistique, l'Harmattan, Paris, 1999.

-NEVEU F., "Détachement et connexité" inSyntaxe et sémantique, n.8, 2007/1, p.p.165-178, mis en ligne sur Cairn.info le 1/10/2016, https: //doi.org/10.3917/ss.008.0165.

-PIRES M., "Usages et stratégies de tutoiement dans l'écrit public" in Langage et société, n. 108, 2004, p.p.27-56, mis en ligne sur Cairn.info le 1/5/2008, https: //doi.org/10.3917/ls.108.0027.

-VINCENT D., "Remarques sur le tutoiement et le vouvoiement en français parlé au Québec", Actes du colloque, La journée du Québec, Institut d'études romanes, université de Copenhague, 2001, p.p. 11-22. 